\title{
Classification Model to Discriminate People with and without Pain in the Lower Back and Lower Limb using Symmetry Data
}

\author{
Si-hyun Kim, PT, Ph.D ${ }^{1}$; Siwoo Jeong, PT, Ph.D ${ }^{2}$; Kyue-nam Park, PT, Ph.D \\ ${ }^{1}$ Department of Physical Therapy, Sangji University, Wonju, South Korea \\ ${ }^{2}$ Department of Biological Sciences, Northern Arizona University, Flagstaff, AZ 86011-5640, USA \\ ${ }^{3}$ Department of Physical Therapy, College of Medical Science, Jeonju University, Jeonju, South Korea
}

Background Multiple factors are associated with lower back and lower limb (LB \& LL) pain, such as impaired muscle strength, balance, endurance, and motor control, and altered movement patterns. Symmetry of motion, strength and balance are goals for rehabilitation in patients with LB \& LL pain. When classifying patients before or during on- and offline assessment, it is necessary that an easy to use functional test be available for clinicians.

Purpose To establish a classification tree model for discriminating people with and without LB \& LL pain during walking using symmetry values from side plank endurance test, hip abductor strength test, one-leg standing time tests and walking tests.

Study design Cross-sectional study

Methods A total of 100 subjects with and without LB \& LL pain during walking participated. We measured the side plank endurance time, hip abductor strength and one-leg standing time with eyes open and closed, and the sagittal and frontal head angles at comfortable and fast walking speeds using a wearable wireless earbud sensor and calculated the symmetry index (SI) for each test. Classification and regression tree analysis with 10 -fold cross validation was used to develop the classification model.

Results The classification tree had $83 \%$ accuracy for discriminating people with and without LB \& LL pain during walking. The most important factor for classification was the SI of the one-leg standing time with eyes closed; the second-most important factor was the SI of the frontal head angle during fast walking.

Conclusions The present classification model can differentiate people with and without LB \& LL pain during walking based on symmetry data acquired during functional tests, such as one-leg standing time with the eyes closed and fast walking test using the wearable device. Based on the present results, clinicians can classify patients before and during on- and offline assessments using cutoff values of the SI of the one-leg standing test with eyes closed of $63.88 \%$, and of frontal head motion during a fast-walking test of $63.31 \%$.

Key words Decision tree; Lower back; Lower limb; Pain; Symmetry.

\section{INTRODUCTION}

Lower limb pain is concomitant with back pain in $61.8 \%$ of cases. ${ }^{1}$ Specifically, pain in the back and hip is significantly related with knee and ankle pain. ${ }^{1}$ Early diagnosis

\author{
J Musculoskelet \\ Sci Technol \\ 2021; 5(2): 72-79 \\ Published Online \\ Dec 31, 2021 \\ pISSN 2635-8573 \\ eISSN 2635-8581 \\ Article History \\ Received 19 Nov 2021 \\ Revised 30 Nov 2021 \\ (1st) \\ Revised 6 Dec 2021 \\ (2nd) \\ Accepted 6 Dec 2021

\section{CONTACT} \\ knpark@jj.ac.kr \\ Kyue-nam Park, \\ Department of Physical \\ Therapy, College of \\ Medical Science, Jeonju \\ University, Jeonju, South \\ Korea \\ This is an Open-Access article \\ distributed under the terms of \\ the Creative Commons Att- \\ ribution Non-Commercial $\mathrm{L}$ \\ cense (hutpt/ereativecomol \\ cense (Attp./Mreativecommons. \\ pericentic \\ permits unrestricted non-co- \\ and reproduction in any me- \\ dium, provided the origina \\ work is properly cited.
}

can prevent recurrence of pain. ${ }^{2}$ A recent review suggested that classification and prediction of pain using artificial intelligence and machine learning algorithms (decision tree, support vector machine, artificial neural network, k-nearest neighbor, etc.) has significant potential. ${ }^{3}$ A classification 
model with $70-100 \%$ accuracy developed by machine learning algorithms could improve lower back pain classification accuracy using data on kinematics, electromyography, gait parameters, acceleration, pain descriptions and behavior, and magnetic resonance imaging. ${ }^{3}$

Given that multiple factors are linked with lower back and lower limb (LB \& LL) pain, such as impaired muscle strength, balance, endurance, and motor control, as well as altered movement patterns, it is necessary to examine patients from multiple perspectives. ${ }^{4,5}$ Especially, altered movement patterns are observed in the frontal plane in people with lower back pain, patellofemoral pain and chronic ankle instability. ${ }^{6-8}$ Asymmetric lateral trunk sway and lateral pelvic tilt are observed during walking in people with knee osteoarthritis. ${ }^{9}$ People with lower back pain show an asymmetrical pelvic pattern in the frontal plane while sitting and standing, which might cause this asymmetrical trunk motion. ${ }^{7}$ People with patellofemoral pain syndrome show excessive lateral trunk motion, hip adduction and knee abduction during squatting and stepping down. ${ }^{10,11}$ Low hip abductor strength while stepping down can lead to excessive trunk lateral sway or knee valgus. ${ }^{12}$ Reduced balance ability, reflected in the one-leg standing time with the eyes open or closed, is another factor in lower back pain and chronic ankle instability. ${ }^{13,14}$

Symmetry of kinematic data, strength and gait parameters has been used as clinical goals, although most people exhibit movement patterns with right dominance due to physiological asymmetries that cause kinematic changes in the trunk and lower extremities. ${ }^{15,16}$ The symmetry index (SI) has been used to quantify symmetry during gait performance. ${ }^{17}$ People with lower back pain exhibit less symmetric kinematic of the trunk and lower limbs during walking than healthy controls, and asymmetric motor control during walking. ${ }^{18,19}$ Asymmetry in the isometric strength of the hip abductor, reflected in poorer balance in the one-leg standing and drop jump tests following anterior cruciate ligament reconstruction relative to healthy controls, is a risk factor for lower extremity injury. ${ }^{20}$

Input data for machine learning models should be easy to collect, so that the large amount of data needed to build classification models can be obtained. ${ }^{21}$ In the present study, to build a classification tree model, we used easy-to-collect input data from simple, functional tests conducted in the clinical setting, such as a walking test using a wearable (earbud) inertial measurement unit (IMU) sensor, a one-leg standing test, a side plank endurance test and a hip abductor strength test. The purpose of the study was to determine whether a classification tree model can discriminate between people with and without LB \& LL pain during walking using symmetry data as the model input (SIs for the side plank endurance test, hip abductor strength test, and one-leg standing test with eyes open and closed, and frontal and sagittal head angles during comfortable and fast walking). In addition, we derived cutoff values for the most important factors for classifying the groups. We hypothesized that the classification tree model would be able to correctly differentiate people with and without LB \& LL pain during walking.

\section{METHODS}

\section{Participants}

Ten to fifteen subjects were needed per variable of interest to ensure an adequate sample size to develop the model. ${ }^{22,23}$ The present study considered eight variables, so 100 subjects were recruited within range of 80 to 120 subjects as suggested by criteria for calculating the sample size. ${ }^{22,23}$ We included young adults (19-30 years old) with and without LB \& LL pain during walking who engaged in regular exercise (1-3 days per week, for 1-3 h per workout). ${ }^{24}$ Subjects were divided into a pain group (visual analogue scale LB \& LL pain score during walking in daily life $\geq 3$ ) and a non-pain group $(<3)$. The exclusion criteria were LB \& LL musculoskeletal pain too severe for the performance of daily activities or completion of the experiments, and any disorder of the vestibular, neurological, cardiopulmonary or psychological system. All subjects consented to participate in this study and provided informed consent. This study was approved by the Institutional Review Board of Jeonju University (JJIRB-210114-HR2021-0113).

\section{Instrumentation}

\section{1) Wireless earbud-type IMU sensor}

While participants walked on a treadmill, we recorded head angle in the sagittal and frontal planes using a wireless earbud-type IMU sensor. A single high-resolution IMU sensor (BNO080; Ceva Technologies, Inc., Rockville, MD, USA) was embedded into a wireless right earbud (QCY-T6; Dongguan Hele Electronics Co., Ltd., China). This sensor was equipped with a triaxial accelerometer and gyroscope to calculate head angle from raw linear acceleration and angular rotation data. The IMU recorded acceleration in the vertical axis while each subject stood. The sampling frequency was $100 \mathrm{~Hz}$. Data obtained from the IMU were transmitted via a Bluetooth antenna to a computer. Prior to the angle calculations, a low-pass filter was used to remove any linear acceleration. Offset calibration was conducted for $1 \mathrm{~s}$ before starting each set of measurements. Data were 
analyzed using a program written in MATLAB (version R2018a; MathWorks, Natick, MA, USA).

\section{2) Tensiometer}

We measured the strength of the hip abductor (in kgf) during maximal isometric contraction using a tensiometer with a non-elastic band (Smart KEMA; Factorial Holdings Co., Seoul, South Korea). The strength measurement system had two load cells. The force of the hip abductor was measured and transmitted to a tablet PC (Galaxy Tab A6 10.1; Samsung Inc., Seoul, South Korea) via a Bluetooth device. Strength data were analyzed using Smart KEMA software (Factorial Holdings Co.).

\section{Procedure}

The experimental procedure consisted of two sessions: baseline measurements and warm-up, and measurement of symmetry. Measurement of four types of symmetry were conducted in a random order, determined by the random function in Excel software. SI, expressed as a percentage, was used to quantify the asymmetry of each variable (side plank "endurance time", strength of the hip abductor, oneleg standing time with eyes open and closed, and sagittal and frontal head angles during treadmill walking at comfortable and fast speeds) using the formula: ${ }^{25}$

$$
\text { SI }[\%]=100 \%-\left(\frac{\text { Left side }- \text { Right side }}{\text { Left side }+ \text { Right side }} \times 100 \%\right)
$$

An SI value of $100 \%$ indicates perfect symmetry; the lower the value, the worse the asymmetry.

\section{1) Baseline measurements and warm-up}

Subject characteristics (age, sex, height, weight, body mass index and LB \& LL pain intensity) were measured at baseline. Subjects performed 5 minutes $(\mathrm{min})$ of indoor cycling to warm up, followed by a 5 -min rest. ${ }^{26}$

\section{2) Measurement of symmetry in the side plank} endurance test

To perform the side plank endurance test, the subjects lay on their side with their legs extended. The subjects were asked to lift their hips off the floor while supported by one elbow and both feet. The goal was to maintain the sides of the trunk and lower legs in a straight line. The subjects were asked to maintain this position for as long as possible; the time was recorded as the measure of plank endurance (Figure 1A). The test ended when a straight line could no longer be maintained, such that the hip dropped to the floor, or the subject asked to stop. Subjects were tested on both sides, resting for $5 \mathrm{~min}$ in between. ${ }^{27}$

\section{3) Measurement of symmetry in the hip abductor} strength test

While in the side-lying position, the maximal isometric strength of the hip abductor was measured as described above. The pelvis is held in position during this test, to confirm whether compensatory pelvic elevation and rotation have occurred. During the test, the subjects were asked to extend the hip and knee on the tested side, with $10^{\circ}$ of hip abduction, while the hip and knee on the non-tested side were flexed slightly to maintain the side-lying position (Figure 1B). The duration of the test was $5 \mathrm{~s}$. Both sides were tested, and the highest force was recorded and used in the data analysis. ${ }^{28}$

4) Measurement of symmetry in the one-leg standing test with eyes open and closed

We measured the one-leg standing times with eyes open and closed for both legs (time between raising the leg and placing it back on the floor) (Figure 1C). ${ }^{29}$

5) Measurement of symmetry of head motion in the treadmill walking test at comfortable and fast speeds

Before performing the treadmill walking test, each subject placed the wireless earbud with the IMU sensor into their right ear, for measurement of head angle in the sagittal and frontal planes. Subjects were asked to look straight ahead. Comfortable and fast speeds were selected by subjects before the data recording began (labeled as "walk comfortably" and "walk as fast as possible without running", respectively) (Figure 1D).$^{30}$ After the treadmill reached the self-selected speed, the tester collected sagittal and frontal head motion data during $1 \mathrm{~min}$ of walking. Only the middle $50 \mathrm{~s}$ of the collected data were analyzed; thus, the initial and last $5 \mathrm{~s}$ of data were excluded. Subjects rested for $1 \mathrm{~min}$ between the comfortable and fast speed conditions.

\section{Statistical analysis}

The data were tested for normality using the ShapiroWilk test. The independent $t$-test was used to compare subject characteristics between the groups with and without LB \& LL pain. Classification and regression tree (CART) analysis was used to develop a classification model for discriminating the groups. The eight classification variables were the SI values of the side plank endurance test, hip abductor strength test, one-leg standing test with eyes open and closed, and the sagittal and frontal head angles during walking at comfortable and fast speeds. A classification tree selected each predictor variable, with impurity minimized 


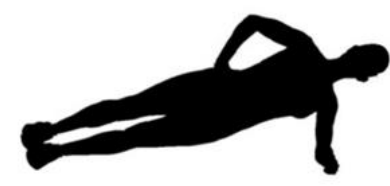

[A]

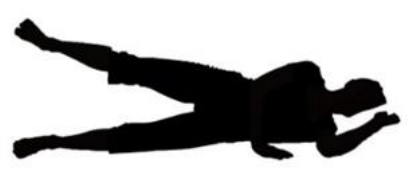

[B]



[C]

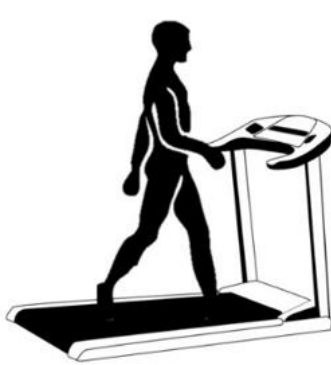

[D]

Figure 1. Symmetry tests (side plank endurance test [A], hip abductor strength test [B], one-leg standing test with eyes open and closed [C], and walking test at comfortable and fast speeds for measuring the sagittal and frontal head angles [D])

using the Gini index. Out-of-sample errors on unseen data were obtained through ten-fold cross validation that was used to avoid overfitting and evaluate the CART model. ${ }^{31}$ All data were randomly split into 10 subsets, 9 of which were used for training the model; the tenth was used evaluating an out-of-sample error. This procedure was repeated for all subsets, such that they were all used as the testing sample. The optimal number of nodes was selected by a backward pruning method. Statistical analyses were performed using SPSS software (ver. 26.0; IBM Corp, Armonk, NY, USA). The significance threshold was set as $p<0.05$.

\section{RESULTS}

There were no significant differences in subject characteristics between the groups except for pain intensity during walking (Table 1). In the pain group, the numbers of subjects with lower back, hip, knee and ankle pain were 3, 6 , 10 and 11 , respectively, including concomitant pain in multiple regions of the body. Figure 2 shows the CART classifications of the subjects with and without LB \& LL pain. The tree has a total of five nodes, of which three are leaf nodes. The most important classification variable was the SI of the one-leg standing test with eyes closed; the second-most important variable was frontal head angle during fast walking. An example of how to interpret the classification tree is as follows: IF the SI value of the oneleg standing time with eyes closed is $\leq 63.88 \%$ AND the SI value of the frontal head angle during fast speed walking is $\leq 63.31 \%$, THEN it is predicted that the participant belongs to the pain group. The overall classification accuracy of the decision tree model was $83 \%$. The confusion matrix is shown in Table 2.

\section{DISCUSSION}

The aim of this study was to determine whether a classification tree model could be used to classify people with and without LB \& LL pain during walking. The developed model had an $83 \%$ accuracy rate based on symmetry data acquired during functional tests, i.e., endurance, strength,

Table 1. Demographic characteristics of the participants

\begin{tabular}{cccc}
\hline Variables & Non-pain group & Pain group & $p$-value \\
\hline Male/female, $\mathrm{n}$ & $43 / 36$ & $6 / 15$ & - \\
\hline Age, years & $22.5 \pm 3.0$ & $22.1 \pm 2.0$ & 0.60 \\
\hline Weight, $\mathrm{kg}$ & $66.2 \pm 13.8$ & $61.8 \pm 15.1$ & 0.21 \\
Height, $\mathrm{cm}$ & $169.4 \pm 8.4$ & $166.0 \pm 8.2$ & 0.11 \\
\hline Body mass index, $\mathrm{kg} / \mathrm{m}^{2}$ & $22.9 \pm 3.3$ & $22.2 \pm 3.8$ & 0.42 \\
\hline Pain intensity (VAS), cm & $0.2 \pm 0.5$ & $3.8 \pm 0.9$ & $<0.01^{*}$ \\
\hline
\end{tabular}

Data are expressed as mean \pm standard deviation. ${ }^{*} p$-value under 0.05 was statistically significant. Abbreviation: VAS, visual analogue scale. 


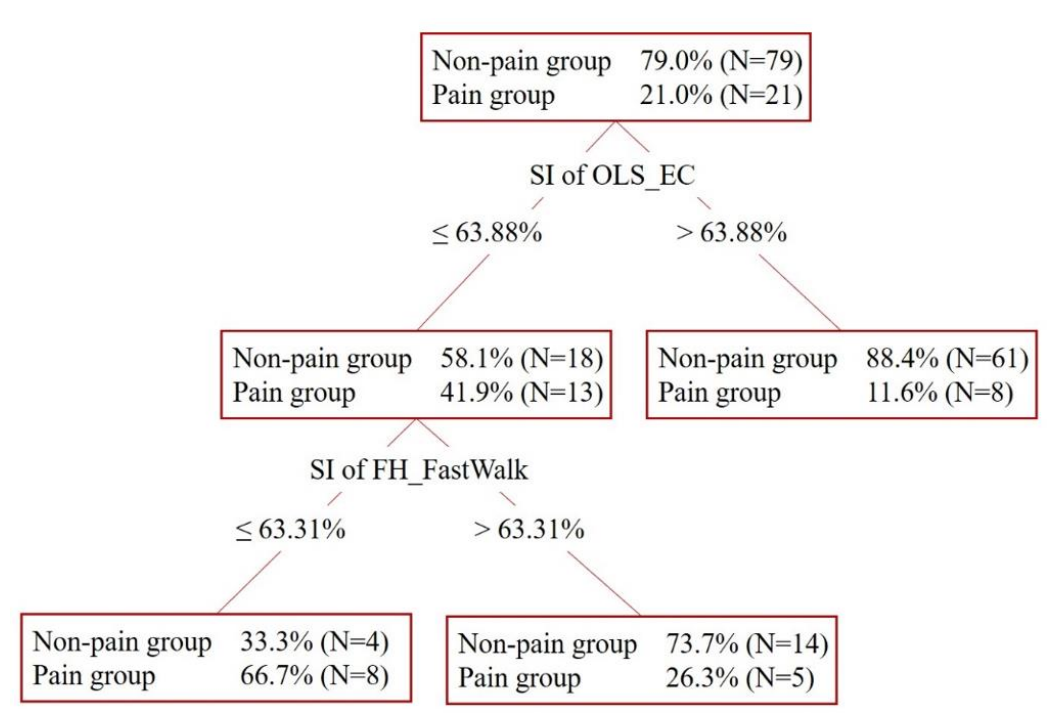

Figure 2. Classification tree model for distinguishing groups with and without lower back and lower limb pain (SI; symmetry index, OLS_EC; one leg standing time with eye closed, FH_FastWalk; frontal head angle during a fastwalking test).

Table 2. Confusion matrix of the classification tree

\begin{tabular}{ccccc}
\hline & $\begin{array}{c}\text { Non-pain } \\
\text { group }\end{array}$ & $\begin{array}{c}\text { Pain } \\
\text { group }\end{array}$ & Accuracy & $\begin{array}{c}\text { Overall } \\
\text { accuracy }\end{array}$ \\
\hline Non-pain group & 75 & 4 & $94.9 \%$ & \\
Pain group & 13 & 8 & $38.1 \%$ & $83.0 \%$ \\
\hline
\end{tabular}

balance and walking tests, performed using a wearable device; such data are easy to collect in clinics. According to our results, when the SI values of the one-leg standing time with eyes closed is $\leq 63.88 \%$ and the frontal head angle during fast walking is $\leq 63.31 \%$, clinicians can pre-classify the patient into the LB \& LL pain group. Whereas, when the SI value of the one-leg standing time with eyes closed is $>63.88 \%$ or SI values of the one-leg standing time with eyes closed is $\leq 63.88 \%$ and the frontal head angle during fast walking is $>63.31 \%$, clinicians can pre-classify the people without the LB \& LL pain group. Using the cutoff values established herein for SI value, the present model can be applied by clinicians for pre-classification of patients before offline examinations and telerehabilitation.

The most important factor for classification was the SI of the one-leg standing time with eyes closed. The standing time was shorter in patients with lower back pain than in healthy controls, possibly because patients with lower back pain have reduced trunk and lumbar proprioception in the absence of visual input. ${ }^{32,33}$ Similarly, people with a history of ankle sprain have reduced somatosensory feedback in the foot and ankle. ${ }^{14}$ People with lower back or ankle pain may tend to depend on visual information to maintain bal- ance. ${ }^{13,14}$ Postural asymmetries in the one-leg stance and drop jump tests following anterior cruciate ligament reconstruction are present compared to matched controls. ${ }^{34}$ The LB \& LL pain group in the present study may have relied more on visual information for balance, leading to asymmetry in the one-leg standing test with eyes-closed condition being the best variable for classification and asymmetry in eye-open condition not being included in the model.

The second-best predictor for classification in this study was the SI of the frontal head angle during fast walking, despite the SI of the sagittal head angle during walking not being included in the model. This result supports previous studies demonstrating altered movement patterns in the frontal plane, but not the sagittal one, in people with LB \& LL pain. ${ }^{6-8}$ Previous studies found that people with chronic ankle instability exhibit excessive lateral trunk displacement towards the stance limb during walking and the star excursion balance test. ${ }^{35,36}$ People with chronic ankle instability use a compensatory strategy characterized by a lateral shift of the center of gravity, with increased force bearing on the side of the foot during the stance phase to overcome external inversion torque while walking. ${ }^{37}$ Fast walking with unilateral leg loading can also induce an asymmetrical gait pattern, ${ }^{38}$ so the SI of the frontal head angle during slow walking not being included in the model. Unilateral trunk sway might induce asymmetry in frontal head motion during fast walking; this could explain the importance of the SI of the frontal rather than sagittal angle in this study.

Our study had some limitations. First, the number of 
subjects with LB \& LL pain was smaller than that without it, although we are satisfied that the sample size was adequate for developing the decision tree model. Secondly, we assessed the symmetry of trunk motion during walking using an earbud-type IMU sensor. Although a sensor on the trunk is more appropriate than one on the head, the ear is a more familiar location for a sensor because of the widespread use of in-ear audio devices. ${ }^{39}$ Also, a previous study suggested that the correlation between head and trunk motions is stronger in people with vestibular hypofunction; ${ }^{40}$ the present study obtained symmetry data indirectly via the head instead of directly via the trunk. To confirm our head motion findings, a further study with a large sample of LB $\&$ LL pain sufferers is needed to develop a classification model based on symmetry data obtained from a trunk rather than head sensor.

\section{CONCLUSIONS}

The present classification model can differentiate people with and without LB \& LL pain during walking based on symmetry data acquired while performing functional tests, such as one-leg standing test with eyes closed and walking tests using a wearable sensor. Based on the present results, clinicians can discriminate before or during on- and offline assessment using cutoff values of the SI of the one-leg standing test with eyes closed of $63.88 \%$, and of frontal head motion during a fast-walking test of $63.31 \%$.

\section{Key Points}

Question Can a classification tree model be established for discriminating people with and without pain in the lower back and lower limb during walking using symmetry data obtained from functional tests?

Findings A classification tree was developed that can discriminate between people with and without pain in the lower back and lower limb during walking with $83 \%$ accuracy. The classification factors were the symmetry indexes of the one-leg standing time with eyes closed and frontal head angle during fast walking.

Meaning Clinicians can apply cutoff values of the symmetry indexes of one-leg standing with eyes closed (63.88\%) and frontal head angle in a fast-walking test $(63.31 \%)$ when conducting pre-screening in on- and offline evaluations.

\section{Article information}

Conflict of Interest Disclosures: None.

Funding/Support: This work was supported by the National Research Foundation of Korea (NRF) grant funded by the Korea government (MSIT) (No. 2020R1A2C210 2729).

Acknowledgment: None.

Ethic Approval: This study was approved by the Institutional Review Board of Jeonju University (JJIRB210114-HR-2021-0113).

\section{REFERENCES}

1. Sogi Y, Hagiwara Y, Yabe Y, et al. Association between trunk pain and lower extremity pain among youth soccer players: a cross-sectional study. BMC Sports Sci. Med. Rehabilitation. 2018;10:13.

2. Al Imran A, Rifat MRI, Mohammad R. Enhancing the classification performance of lower back pain symptoms using genetic algorithm-based feature selection. Proc. Int. Jt. Conf. Comput. Intell. Springer, Singapore, 2020; 455-469.

3. Tagliaferri SD, Angelova M, Zhao X, et al. Artificial intelligence to improve back pain outcomes and lessons learnt from clinical classification approaches: three systematic reviews. NPJ Digit Med. 2020;3:93.

4. Rum L, Brasiliano P, Vannozzi G, Laudani L, Macaluso A. Non-specific chronic low back pain elicits kinematic and neuromuscular changes in walking and gait termination. Gait Posture. 2021;84:238-244.

5. Rahimi A, Arab AM, Nourbakhsh MR, Hosseini SM, Forghany S. Lower limb kinematics in individuals with chronic low back pain during walking. $J$ Electromyogr Kinesiol. 2020;51:102404.

6. Glaviano NR, Saliba S. Association of altered frontal plane kinematics and physical activity levels in females with patellofemoral pain. Gait Posture. 2018;65:86-88.

7. Al-Eisa E, Egan D, Deluzio K, Wassersug R. Effects of pelvic asymmetry and low back pain on trunk kinematics during sitting: a comparison with standing. Spine. 2006; 31(5):E135-143.

8. Kim H, Son SJ, Seeley MK, Hopkins JT. Altered movement strategies during jump landing/cutting in patients with chronic ankle instability. Scand J Med Sci Sports. 2019;29(8):1130-1140.

9. Hunt MA, Wrigley TV, Hinman RS, Bennell KL. Individuals with severe knee osteoarthritis (OA) exhibit altered proximal walking mechanics compared with individuals with less severe OA and those without knee pain. Arthritis Care Res (Hoboken). 2010;62(10):14261432.

10. Lim EH, Kim ME, Kim SH, Park KN. Effects of posterior $\mathrm{X}$ taping on movement quality and knee pain intensity during forward-step-down in patients with 
patellofemoral pain syndrome. J Sports Sci Med. 2020; 19(1):224-230.

11. Kim JS, Kim YW, Woo YK, Park KN. Validity of an artificial intelligence-assisted motion-analysis system using a smartphone for evaluating weight-bearing activities in individuals with patellofemoral pain syndrome. J Musculoskelet Sci Technol. 2021;5(1):34-40.

12. Almeida GP, Silva AP, França FJ, Magalhães MO, Burke TN, Marques AP. Relationship between frontal plane projection angle of the knee and hip and trunk strength in women with and without patellofemoral pain. J Back Musculoskelet Rehabil. 2016;29(2):259266.

13. Mok NW, Brauer SG, Hodges PW. Hip strategy for balance control in quiet standing is reduced in people with low back pain. Spine. 2004;29(6):E107-112.

14. Song K, Burcal CJ, Hertel J, Wikstrom EA. Increased visual use in chronic ankle instability: a meta-analysis. Med Sci Sports Exerc. 2016;48(10):2046-2056.

15. Lewek M, Rudolph K, Axe M, Snyder-Mackler L. The effect of insufficient quadriceps strength on gait after anterior cruciate ligament reconstruction. Clin Biomech (Bristol, Avon). 2002;17(1):56-63.

16. Sadeghi H, Allard P, Prince F, Labelle H. Symmetry and limb dominance in able-bodied gait: a review. Gait Posture. 2000;12(1):34-45.

17. Radebold A, Cholewicki J, Panjabi MM, Patel TC. Muscle response pattern to sudden trunk loading in healthy individuals and in patients with chronic low back pain. Spine. 2000;25(8):947-954.

18. Sung PS, Danial P. A kinematic symmetry index of gait patterns between older adults with and without low back pain. Spine. 2017;42(23):E1350-e1356.

19. Tawfik B. Symmetry and linearity of trunk function in subjects with non-specific low back pain. Clin Biomech (Bristol, Avon). 2001;16(2):114-120.

20. De Blaiser C, Roosen P, Willems T, et al. The role of core stability in the development of non-contact acute lower extremity injuries in an athletic population: a prospective study. Phys Ther Sport. 2021;47:165-172.

21. Fan C, Sun Y, Xiao F, et al. Statistical investigations of transfer learning-based methodology for short-term building energy predictions. Applied Energy. 2020;262: 114499.

22. McGinn TG, Guyatt GH, Wyer PC, Naylor CD, Stiell IG, Richardson WS. Users' guides to the medical literature: XXII: how to use articles about clinical decision rules. Evidence-Based Medicine Working Group. Jama. 2000;284(1):79-84.

23. Wasson JH, Sox HC, Neff RK, Goldman L. Clinical prediction rules. Applications and methodological standards. N Engl J Med. 1985;313(13):793-799.

24. Butowicz CM, Ebaugh DD, Noehren B, Silfies SP. Validation of two clinical measures of core stability. Int J Sports Phys Ther. 2016;11(1):15-23.

25. Agrawal V, Gailey R, O'Toole C, Gaunaurd I, Dowell T. Symmetry in external work (SEW): a novel method of quantifying gait differences between prosthetic feet. Prosthet Orthot Int. 2009;33(2):148-156.

26. Hughes S, Chapman DW, Haff GG, Nimphius S. The use of a functional test battery as a non-invasive method of fatigue assessment. PloS one. 2019;14(2):e0212870.

27. McGill SM, Childs A, Liebenson C. Endurance times for low back stabilization exercises: clinical targets for testing and training from a normal database. Arch Phys Med Rehabil. 1999;80(8):941-944.

28. Jeon IC. Comparisons of test-retest reliability of strength measurement of gluteus medius strength between break and make test in subjects with pelvic drop. J Kor Phys Ther. 2019;31(3):147-150.

29. Rikli R, Busch S. Motor performance of women as a function of age and physical activity level. J Gerontol. 1986;41(5):645-649.

30. Wrightson JG, Schäfer L, Smeeton NJ. Dual-task prioritization during overground and treadmill walking in healthy adults. Gait Posture. 2020;75:109-114.

31. Kumar N, ReddyY G, Chatterji S, Prasad J, Sarkar D. An application of classification and regression tree on qualitative soil survey data for land capability classification. J Soil Water Conservation. 2013;12(4):301306.

32. Radebold A, Cholewicki J, Polzhofer GK, Greene HS. Impaired postural control of the lumbar spine is associated with delayed muscle response times in patients with chronic idiopathic low back pain. Spine. 2001; 26(7):724-730.

33. Brumagne S, Cordo P, Lysens R, Verschueren S, Swinnen S. The role of paraspinal muscle spindles in lumbosacral position sense in individuals with and without low back pain. Spine. 2000;25(8):989-994.

34. Mohammadi F, Salavati M, Akhbari B, Mazaheri M, Khorrami M, Negahban H. Static and dynamic postural control in competitive athletes after anterior cruciate ligament reconstruction and controls. Knee Surg Sports Traumatol Arthrosc. 2012;20(8):1603-1610.

35. Hoch MC, Gaven SL, Weinhandl JT. Kinematic predictors of star excursion balance test performance in individuals with chronic ankle instability. Clin Biomech (Bristol, Avon). 2016;35:37-41.

36. Abdelraouf OR, Abdel-Aziem AA. Ankle and foot 
mechanics in individuals with chronic ankle instability during shod walking and barefoot walking: a crosssectional study. Chin J Traumatol. 2021;24(3):174-179.

37. Riemann BL. Is there a link between chronic ankle instability and postural instability?. J Athl Train. 2002; 37(4):386-393.

38. Kodesh E, Kafri M, Dar G, Dickstein R. Walking speed, unilateral leg loading, and step symmetry in young adults. Gait Posture. 2012;35(1):66-69.

39. Tavakoli Golpaygani A, Mehdizadeh AR. Future of wearable health devices: smartwatches VS smart headphones. J Biomed Phys Eng. 2021;11(5):561-562.

40. Zhang F, Liu P, Ou Y, et al. Trunk and head control during walking in patients with unilateral vestibular hypofunction: effect of lower limb somatosensory input. Am J Phys Med Rehabil. 2019;98(10):906-913. 